\title{
Acute effects of coffee on skin blood flow and microvascular function
}

Erik Tesselaar, Dzeneta Nezirevic Dernroth and Simon Farnebo

The self-archived postprint version of this journal article is available at Linköping University Institutional Repository (DiVA):

http:/ / urn.kb.se/ resolve?urn=urn:nbn:se:liu:diva- 145382

N.B.: When citing this work, cite the original publication.

Tesselaar, E., Nezirevic Dernroth, D., Farnebo, S., (2017), Acute effects of coffee on skin blood flow and microvascular function, Microvascular Research, 114, 58-64.

https:// doi.org/ 10.1016/j.mvr.2017.06.006

Original publication available at:

https:/ / doi.org/ 10.1016/j.mvr.2017.06.006

Copyright: Elsevier

http:// www.elsevier.com/

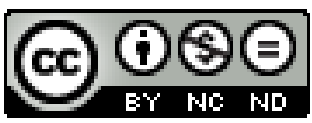




\section{Acute effects of coffee on skin blood flow and microvascular function}

Erik Tesselaar $^{1,2}$, Dzeneta Nezirevic Dernroth ${ }^{1,3}$, Simon Farnebo ${ }^{1,4}$

1. Department of Clinical and Experimental Medicine, Faculty of Health Sciences, Linköping University, Linköping, Sweden.

2. Department of Radiation Physics, Department of Medical and Health Sciences, Linköping University, Linköping, Sweden.

3. Department of Clinical Chemistry, Region Östergötland, Linköping, Sweden.

4. Department of Plastic Surgery, Hand Surgery, and Burns, Linköping University, Linköping, Sweden.

\section{Corresponding author:}

Erik Tesselaar, Assoc. Prof

Department of Clinical and Experimental Medicine

Faculty of Health Sciences

Linköping University

SE-58185 Linköping, Sweden

Phone: + $4610-1038059$

E-mail: erik.tesselaar@liu.se

Abbreviations: SD: standard deviation, BMI: body mass index, ACh: acetylcholine chloride, SNP: sodium nitroprusside, LSCI: laser speckle contrast imaging, LDF: laser Doppler flowmetry, HPLC: high performance liquid chromatography

Word count: 6221 words 


\section{Abstract}

\section{Objective}

Studies on the acute effects of coffee on the microcirculation have shown contradicting results. This study aimed to investigate if intake of caffeine-containing coffee changes blood flow and microvascular reactivity in the skin.

\section{Methods}

We measured acute changes in cutaneous vascular conductance (CVC) in the forearm and the tip of the finger, the microvascular response to transdermal iontophoresis of acetylcholine (ACh) and sodium nitroprusside (SNP) and post-occlusive reactive hyperemia (PORH) in the skin, after intake of caffeinated or decaffeinated coffee.

\section{Results}

Vasodilatation during iontophoresis of ACh was significantly stronger after intake of caffeinated coffee compared to after intake of decaffeinated coffee $(1.26 \pm 0.20 \mathrm{PU} / \mathrm{mmHg}$ vs $1.13 \pm 0.38 \mathrm{PU} / \mathrm{mmHg}, \mathrm{P}<0.001)$. Forearm CVC before and after PORH were not affected by caffeinated and decaffeinated coffee. After intake of caffeinated coffee, a more pronounced decrease in CVC in the fingertip was observed compared to after intake of decaffeinated coffee (-1.36 PU/mmHg vs. -0.52 PU/mmHg, $\mathrm{P}=0.002)$.

\section{Conclusions}

Caffeine, as ingested by drinking caffeinated coffee acutely improves endothelium-dependent microvascular responses in the forearm skin, while endothelium-independent responses to PORH and SNP iontophoresis are not affected. Blood flow in the fingertip decreases 
markedly during the first hour after drinking caffeinated coffee compared to decaffeinated coffee.

\section{Keywords}

coffee, caffeine, skin, microcirculation, laser Doppler flowmetry, laser speckle contrast imaging 


\section{Introduction}

Coffee is one of the most widely consumed beverages in the world. Although it is well known to be a major source of caffeine, coffee also contains a wide range of compounds that are not all completely identified and of which the effects on the human body is yet unknown. Most studies find that regular coffee intake increases blood pressure slightly and raises plasma cholesterol and homocysteine levels [1], whereas coffee consumption is associated with a reduced incidence of type 2 diabetes mellitus [2], a decrease in inflammatory markers [3] and improved endothelial function [4]. Caffeine consumption also affects pain modulation [5] and interacts with skin carcinomas, with some evidence pointing at a protective effect of caffeine on malignant melanoma [6,7].

Prospective studies have generally not shown a consistent positive association between coffee intake and cardiovascular diseases, with some results even suggesting protective effects [810]. Studies on acute vascular effects of caffeine have, however, shown contradictory results, with most studies showing a slight increase elevated blood pressure and arterial stiffness [11,12], decreased glucose disposal [13], and impaired endothelial function [14,15]. On the other hand, some studies have found that caffeine causes an increase in endothelium dependent vasodilatation [16-18]. The reason for these conflicting findings may reside in the differences in study methods, study population and the presence of other compounds in coffee besides caffeine, such as some phenolic components, which are well known to have a strong antioxidant capacity. It is thus possible that this antioxidant capacity compensates for the negative effects of caffeine $[19,20]$.

The microcirculation is crucial in the regulation of vascular resistance and the blood flow in tissues and critical organs. Because the skin is easily accessible, its microvascular bed is has 
become a popular model for studying microvascular function. The cutaneous microcirculation can be investigated noninvasively using physiological and drug provocations that specifically target vascular signaling pathways. For instance, endothelium-dependent microvascular responses can be studied using iontophoresis of acetylcholine (ACh), often combined with iontophoresis of sodium nitroprusside (SNP) as an endothelium-independent control. Postocclusive reactive hyperemia (PORH) in the skin can be used to study the involvement of sensory nerves and hyperpolarizing mechanisms. Importantly, the recent development of image-based blood flow measurement techniques has further improved the reproducibility of microvascular assessment in the skin [21,22].

In this double blind, randomized, placebo-controlled cross-over study, we therefore aimed to investigate if intake of caffeine-containing coffee, or decaffeinated coffee changes blood flow and microvascular reactivity in the skin. We specifically studied (1) changes in basal cutaneous vascular conductance (CVC) in the forearm and the tip of the finger using noninvasive, optical measurement techniques, (2) the involvement of the endothelium using transdermal iontophoresis of acetylcholine (ACh) and sodium nitroprusside (SNP), and (3) the involvement of sensory nerves and hyperpolarizing mechansisms using post-occlusive reactive hyperemia (PORH) following 5-minutes of forearm arterial occlusion. 


\section{Materials and Methods}

\section{Subjects}

Sixteen normotensive, non-smoking subjects (8 female, 8 male) without any known cardiovascular or skin diseases, mean (standard deviation, SD) age 23.4 (2.3) years were included in the study after they had given informed and written consent. Smokers and subjects with a blood pressure above 140/90 mmHg were excluded, as microvascular function is impaired by cigarette smoking and correlated to blood pressure [23,24]. The study subjects had a mean weight of 76.9 (11.6) kg, a mean length of 177 (8) cm and a mean BMI of 24.4 (2.7) $\mathrm{kg} / \mathrm{m}^{2}$. Before the start of the experiments the subjects, who were regular coffee drinkers, were asked to refrain from eating and drinking any caffeine containing products, including coffee, tea, energy drinks and chocolate for at least 24 hours. They were also asked to avoid strenuous exercise on the day of the experiment. The study was approved by the regional ethics committee in Linköping, Sweden (DNr 2016-119/32), and was done according to the declaration of Helsinki.

\section{Protocol}

A randomized, double-blind, placebo-controlled crossover study was designed. The subjects visited the research lab on two separate visits with at least 5 days and at most 9 days in between. The subjects were randomized to either ingest caffeinated or decaffeinated coffee on their first visit. All measurements took place in the same room, at an ambient temperature of $21 \pm 1^{\circ} \mathrm{C}$. A schematic overview of the experimental protocol is shown in Figure 1 . 


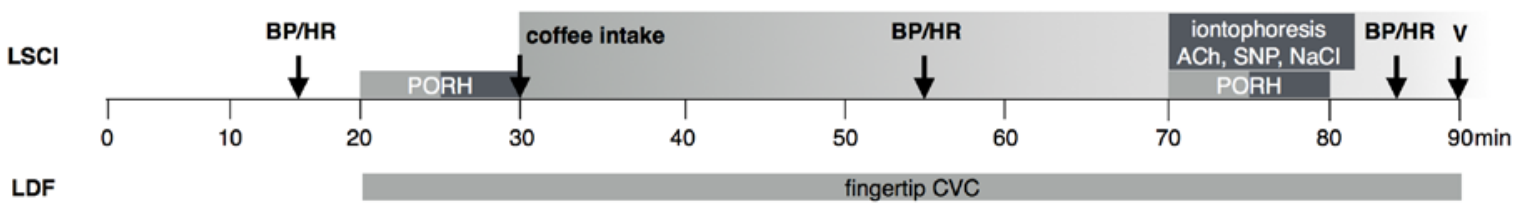

Figure 1. Overview of the experimental protocol. Either caffeinated or decaffeinated coffee was consumed in a double-blind, randomized manner. PORH was done in the right arm, while iontophoresis was done in the left arm. Fingertip cutaneous vascular conductance (CVC) was measured in the left middle finger using a thermostatic laser Doppler probe at $30{ }^{\circ}$ C. LSCI: Laser Speckle Contrast Imaging, LDF: Laser Doppler Flowmetry, BP/HR: blood pressure and heart rate measurement, $P O R H$ : post-occlusive reactive hyperemia, $V$ : venous blood sampling.

Subjects were placed in semi-supine position with their arms at heart level comfortably supported by pillows. A laser Doppler flowmetry (LDF) probe was mounted to the skin of the palmar aspect of the distant phalanx of the third finger of the left hand using double adhesive tape. This LDF probe was used to measure skin perfusion continuously throughout the experiment. After 15 minutes of acclimatization, baseline measurements of blood pressure and heart rate were made. The skin of the volar side of both forearms was gently cleaned with chlorhexidine ethanol (5 mg/mL, Fresenius AB, Uppsala, Sweden). Then, baseline skin perfusion measured in a $5 \times 10 \mathrm{~cm}^{2}$ area on the medial, volar side of the right forearm was for 1 minute using laser speckle contrast imaging (LSCI). Total arterial occlusion was then induced and maintained during 5 minutes using a blood pressure cuff around the upper arm inflated to a pressure of $220 \mathrm{mmHg}$. After the blood pressure cuff was released, the PORH response was recorded until the perfusion had decreased to a stable level of at least $50 \%$ of the maximum level (typically 5 minutes after release of the cuff).

A cup of caffeinated or decaffeinated coffee (Vivalto Lungo or Vivalto Lungo Decaffeinato, Nespresso AB, Stockholm, Sweden) was prepared and consumed by the subject. Neither sugar nor milk was added. Two iontophoresis electrodes were mounted on the volar side of the left forearm, approximately $5 \mathrm{~cm}$ apart, and connected to the current controller. One iontophoresis electrode was filled with ACh and another was filled with either $\mathrm{NaCl}(\mathrm{N}=6)$ or SNP $(\mathrm{N}=10)$. Forty minutes after coffee intake, the drugs were delivered simultaneously using 
a 10-minute, $0.02 \mathrm{~mA}$ anodal (ACh) and cathodal ( $\mathrm{NaCl}$ or SNP) current. Immediately after the start of the iontophoresis, the PORH measurement was repeated on the other arm using the same protocol as before. Finally, at the end of the experiment, blood pressure was measured a second time.

\section{Equipment}

Transdermal iontophoresis was used to deliver ACh, SNP and sodium chloride $(\mathrm{NaCl})$ to the skin, using an iontophoresis current controller (Prion 382, Perimed AB, Järfälla, Sweden) and transparent, ring-shaped drug delivery electrodes (LI 611, Perimed AB, Järfälla, Sweden). Drug delivery electrodes were always placed on the volar side of the left forearm and care was taken to avoid visible veins and birthmarks.

A Laser Speckle Contrast imager (Pericam PSI System, Perimed AB, Järfälla, Sweden) was used to measure perfusion in the skin of forearm during microvascular provocations. The system uses a divergent laser beam with a wavelength of $785 \mathrm{~nm}$. Perfusion images were acquired every two seconds by averaging data from 42 image frames taken in rapid succession (21 frames/seconds). The image size was set to correspond to a $7 \mathrm{~cm} \mathrm{x} 7 \mathrm{~cm}$ area of skin and the spatial resolution of the perfusion image was $0.2 \mathrm{~mm} / \mathrm{pixel}$ at a measurement distance of $20 \mathrm{~cm}$. The system was calibrated according to the manufacturer recommendations. Perfusion images were further analyzed by calculating mean perfusion levels in regions of interest using PIMsoft 1.3 (Perimed AB, Järfälla, Sweden).

A laser Doppler perfusion monitor (Periflux 5000, Perimed AB, Järfälla, Sweden) was used to measure changes in skin perfusion in the fingertip. A thermostatic laser Doppler probe (Probe 457, Perimed AB, Järfälla, Sweden) was used, and was set to a constant temperature of $30{ }^{\circ} \mathrm{C}$. The probe has a fiber separation of $0.25 \mathrm{~mm}$ and measures perfusion at depth of 
approximately $0.5 \mathrm{~mm}$. Perfusion values were measured at a sample rate of 33 recordings per second and were further processed using the manufacturer's software (Perisoft 2.5.5, Perimed AB, Järfälla, Sweden). An automatic sphygmomanometer (M6 Comfort, Omron Healthcare, Hoofddorp, The Netherlands) was used to measure blood pressure and heart rate.

\section{Coffee}

Caffeinated and decaffeinated coffee was prepared from capsules (Vivalto Lungo and Vivalto Lungo Decaffeinato, Nespresso AB, Stockholm, Sweden) using a commercial coffee maker (Krups Inissia, Nespresso AB, Stockholm, Sweden) shortly before intake on the day of the experiment. The caffeinated coffee contains $78 \mathrm{mg}$ of caffeine per capsule whereas the decaffeinated coffee contains 0.065-0.078 mg of caffeine. The decaffeinated coffee was produced from the same type of unroasted coffee beans as the caffeinated coffee, using the Swiss water method, which removes over $99.9 \%$ of the caffeine while preserving the other ingredients in the beans [25].

\section{Drugs}

Acetylcholine chloride (Miochol-E, Novartis Healthcare, Denmark) and sodium chloride, 9 mg/mL (Baxter Medical AB, Kista, Sweden), were obtained from the hospital pharmacy. Sodium nitroprusside dihydrate and caffeine were obtained from Sigma Aldrich (St. Louis, MO, USA). Drugs were dissolved in sterile water to a final concentration of $10 \mathrm{mg} / \mathrm{mL}$. All drug solutions were prepared on the day of the experiments.

\section{Caffeine analysis}

The caffeine analysis in plasma was performed by high performance liquid chromatography (HPLC). HPLC analysis was carried out using Waters Alliance 2695 instrument (Milford, 
MA, USA). Chromatographic separation was obtained on a $75 \mathrm{~mm} \times 3 \mathrm{~mm}(2.5 \mu \mathrm{m}$ particle size) XBridge BEH-C 18 column (Waters, Milford, MA, USA) at room temperature. Detection was made using a 996 photodiode array (PDA) detector (Waters, Milford, MA, USA). Data was collected and analyzed with the Chromeleon data system (version 7).

Stock standard solutions of caffeine were prepared in distilled water. Working standard solutions were prepared by subsequent dilution of stock standard solutions to obtain concentrations of $0,0.5,1,2.5,5$ and $10 \mu \mathrm{g} / \mathrm{mL}$. Blood samples were collected in heparinized glass tubes and centrifuged for 5 min at 2000 G. $200 \mu \mathrm{L}$ plasma was transferred into Eppendorf vials, after which $500 \mu \mathrm{L}$ acetonitrile was added and the sample was mixed on a vortex mixer for $10 \mathrm{~s}$ and centrifuged at $1000 \mathrm{G}$ for $10 \mathrm{~min}$. The supernatant was then evaporated to dryness on a water bath at $35{ }^{\circ} \mathrm{C}$ in a gentle stream of dry nitrogen for 45 min. A mixture of $90 \%$ ammonium phosphate and $10 \%$ acetonitrile was added to vials and the solution was mixed on a vortex mixer for 30 s. $5 \mu$ l of the solution was injected onto the HPLC system.

The mobile phases consisted of (A) $0.05 \mathrm{M}$ ammonium phosphate monobasic, $\mathrm{pH} 4.5$ and (B) acetonitrile. Separation was achieved by multistep gradient elution. The start composition was $93 \% \mathrm{~A}$ and $7 \% \mathrm{~B}$ for $0.5 \mathrm{~min}$ followed by linear increase to $12 \% \mathrm{~B}$ for $4.5 \mathrm{~min}$, and to $50 \%$ B for 4 min. The content of acetonitrile was then linearly increased to $98 \%$ B for 0.5 min and after $1 \mathrm{~min}$ dropped to the starting composition. The flow rate was $0.3 \mathrm{~mL} / \mathrm{min}$ and the injection volume was $5 \mu \mathrm{L}$. Separation was monitored at $230 \mathrm{~nm}$. Column and sampler temperatures were set at room temperature. 


\section{Data analysis}

All data in text and tables are presented as mean (SD). Cutaneous vascular conductance (CVC) was calculated as perfusion, as measured using LSCI and LDF, divided by mean arterial pressure. Two-way analyses of variance (ANOVA) for repeated measures with Sidak's multiple comparisons test were used to test whether changes in blood pressure, heart rate, and CVC were significantly different after drinking caffeinated or decaffeinated coffee. The responses to PORH and iontophoresis were analyzed using baseline and maximum CVC as covariates, whereas fingertip CVC was analyzed before intake of coffee, and at 4 time intervals between 0 and 60 minutes after intake of coffee. A paired Student's t-test was used to compare plasma caffeine concentrations. GraphPad Prism version 6.0 for Mac OS X (GraphPad Software, San Diego, CA, USA, “'http://www.graphpad.com’’) was used for all statistical calculations. For all analyses, probabilities of $<0.05$ were accepted as significant. 


\section{Results}

\section{decaffeinated caffeinated}

\section{Before intake}

Systolic blood pressure (mmHg)

$\begin{array}{ll}115 \pm 13 & 118 \pm 14 \\ 73 \pm 9 & 72 \pm 8 \\ 73 \pm 12 & 69 \pm 12\end{array}$

Heart rate (bpm)

$73 \pm 12$

$0.38 \pm 0.07$

baseline

$0.42 \pm 0.09$

$1.21 \pm 0.24$

PORH

$1.26 \pm 0.18$

Fingertip CVC (PU/mmHg)

absolute CVC

$2.11 \pm 1.20$

$2.64 \pm 1.40$

\section{After intake}

Systolic blood pressure (mmHg)

$$
\begin{aligned}
& 30 \mathrm{~min} \\
& 60 \mathrm{~min}
\end{aligned}
$$

$112 \pm 12$

$116 \pm 15$

Diastolic blood pressure (mmHg)

$$
\begin{aligned}
& 30 \mathrm{~min} \\
& 60 \mathrm{~min}
\end{aligned}
$$$$
110 \pm 12^{\dagger}
$$$$
114 \pm 14
$$

Heart rate (bpm)

$$
\begin{aligned}
& 30 \mathrm{~min} \\
& 60 \mathrm{~min}
\end{aligned}
$$

P-caffeine $(\mu \mathrm{g} / \mathrm{mL})$

$$
71 \pm 9
$$$$
70 \pm 8
$$

$70 \pm 8$

$70 \pm 7$

Forearm CVC (PU/mmHg)

$\begin{array}{lll}\text { baseline } & 0.41 \pm 0.10 & 0.39 \pm 0.08 \\ \text { PORH } & 1.31 \pm 0.20 & 1.26 \pm 0.19 \\ \text { sodium chloride } & 0.31+0.06 & 0.57 \pm 0.27 \\ \text { acetylcholine } & 1.13 \pm 0.38 & \mathbf{1 . 2 6} \pm \mathbf{0 . 2 0} * * * \\ \quad \text { sodium nitroprusside } & 0.99 \pm 0.29 & 1.09 \pm 0.29 \\ \text { Fingertip CVC (PU/mmHg) } & & \\ \text { absolute CVC } & 1.59 \pm 1.34 & 1.28 \pm 1.09 \\ \text { change from before intake } & -0.52 \pm 0.97 & \mathbf{- 1 . 3 6} \pm \mathbf{1 . 1 9 * *}\end{array}$

Table 1. Cardiovascular and microvascular measures before and after intake of either decaffeinated (0.065$0.078 \mathrm{mg}$ ) or caffeinated $(78 \mathrm{mg}$ ) coffee. * indicates $P<0.05$, ** $P<0.01$, *** $P<0.001$ between subjects ingesting caffeinated and decaffeinated coffee. $\dagger$ indicates a significant change from before intake $(P<0.05)$. 


\section{Plasma caffeine levels}

One hour after intake of caffeinated coffee, plasma caffeine levels were significantly higher compared to those measured after intake of decaffeinated coffee $(1.26 \pm 0.29 \mu \mathrm{g} / \mathrm{mL}$ and 0.21 $\pm 0.22 \mu \mathrm{g} / \mathrm{mL}$, respectively, $\mathrm{P}<0.001)$.

\section{Blood pressure and heart rate}

At baseline, there were no significant differences in systolic or diastolic blood pressure or heart rate between the subjects who would ingest caffeinated coffee and those who would ingest decaffeinated coffee (Table 1). Although analysis of variance found no significant interaction between blood pressure or heart rate and intake of caffeinated or decaffeinated coffee, systolic blood pressure was decreased significantly 60 minutes after intake of decaffeinated coffee (baseline: $115 \pm 13$ mmHg; 30 min after intake: $112 \pm 12$ mmHg; 60 minutes after intake: $110 \pm 12 \mathrm{mmHg} ; \mathrm{P}=0.04$. After intake of caffeinated coffee, there was no significant change in systolic or diastolic blood pressure or heart rate.

\section{Iontophoresis of ACh, SNP and NaCl}

At the beginning of iontophoresis, there were no differences in CVC after intake of caffeinated coffee and decaffeinated coffee. Iontophoresis and ACh, SNP and $\mathrm{NaCl}$ all caused a significant increase in CVC (Table 1, Figure 2). However, the response to iontophoresis of ACh was significantly stronger after intake of caffeinated coffee compared to the response after intake of decaffeinated coffee (caffeinated: $1.26 \pm 0.20$ PU/mmHg, decaffeinated: $1.13 \pm$ $0.38 \mathrm{PU} / \mathrm{mmHg}, \mathrm{P}_{\text {time*coffee }}<0.001$ ), while no difference in responses was seen after iontophoresis of SNP (caffeinated: $1.09 \pm 0.29 \mathrm{PU} / \mathrm{mmHg}$, decaffeinated: $0.99 \pm 1.09$ $\mathrm{PU} / \mathrm{mmHg}, \mathrm{P}_{\text {time*}{ }^{*} \text { coffee }}=0.28$ ). No difference in responses was seen after iontophoresis of 
$\mathrm{NaCl}$ (caffeinated: $0.57 \pm 0.27 \mathrm{PU} / \mathrm{mmHg}$, decaffeinated: $0.31 \pm 0.06 \mathrm{PU} / \mathrm{mmHg}, \mathrm{P}_{\text {time*coffee }}=$ $0.58)$.
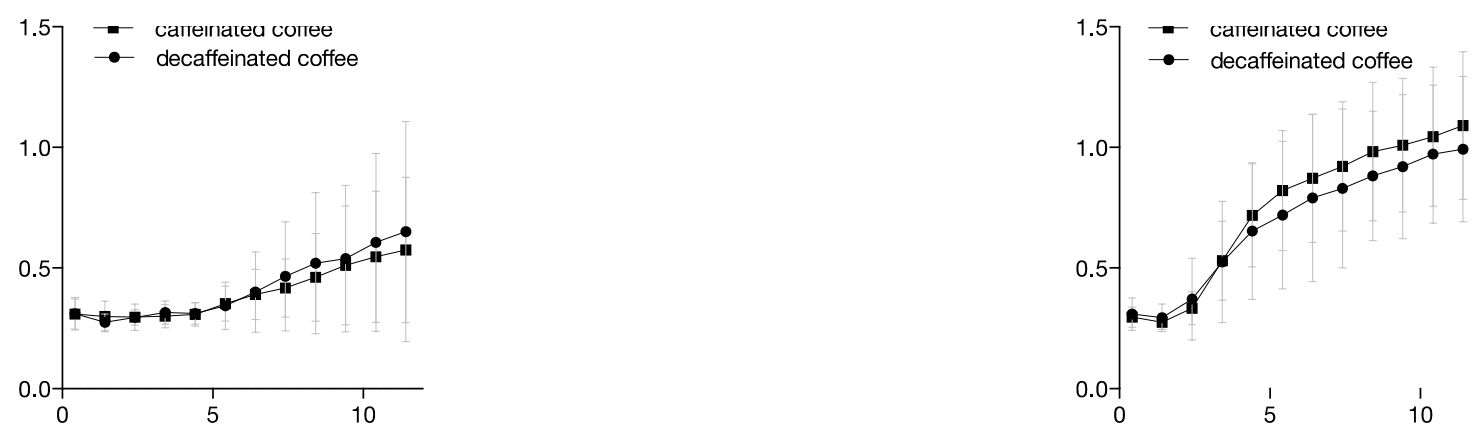

Figure 2. Microvascular response to iontophoresis if $\mathrm{NaCl}(\mathrm{left}, n=6$ ), ACh (middle, $n=16$ ) and SNP (right, $n=10$ ) after intake of caffeinated or decaffeinated coffee. Intake of caffeinated coffee significantly enhanced the increase in cutaneous vascular conductance (CVC) after iontophoresis of ACh $(P<0.001)$. No significant difference in responses were seen with $\mathrm{NaCl}(P=0.99)$ and $\operatorname{SNP}(P=0.07)$.

\section{Forearm CVC and PORH response}

There were no significant differences in forearm CVC between the subjects who ingested caffeinated coffee and those who ingested decaffeinated coffee (Table 1), neither at baseline $(P=0.66)$ nor 45 minutes after intake of coffee $(P=0.81)$. Neither were there any significant differences between the PORH responses before $(\mathrm{P}=0.47)$ nor 45 minutes after intake of coffee $(P=0.31)$.

\section{Fingertip CVC}

There were large variations in absolute CVC in the fingertip between subjects, both at baseline $(2.11 \pm 1.20 \mathrm{PU} / \mathrm{mmHg}$ and $2.64 \pm 1.40 \mathrm{PU} / \mathrm{mmHg}$ in the decaffeinated and caffeinated groups, respectively) and after intake of coffee. Both after intake of caffeinated and decaffeinated coffee, fingertip CVC decreased with time (Figure 3). However, the decrease in CVC was significantly stronger between 30 and 45 minutes (-1.05 PU/mmHg vs. - 
0.29 PU/mmHg, $\mathrm{P}=0.005)$ and between 45 and 60 minutes $(-1.36 \mathrm{PU} / \mathrm{mmHg}$ vs. -0.52

$\mathrm{PU} / \mathrm{mmHg}, \mathrm{P}=0.002$ ) after intake of caffeinated coffee, compared with the decrease after intake of decaffeinated coffee.

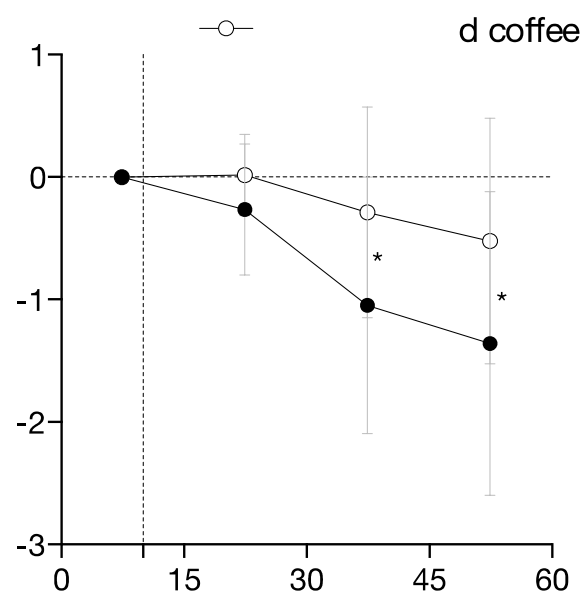

Figure 3. Decrease in cutaneous vascular conductance (CVC) compared to baseline (15-minute period before coffee intake) in the tip of the left middle finger $(n=16)$ after intake of coffee (filled circles) or decaffeinated coffee (open circles), as measured with laser Doppler flowmetry. The decrease in CVC was markedly stronger after intake of caffeinated coffee compared to the decrease in CVC after intake of decaffeinated coffee (-1.05 PU/mmHg vs. $-0.29 \mathrm{PU} / \mathrm{mm} \mathrm{Hg}, P=0.005$, between 30 and 45 minutes after intake, and $-1.36 \mathrm{PU} / \mathrm{mmHg}$ vs. $-0.52 \mathrm{PU} / \mathrm{mmHg}, P=0.002)$ between 45 and 60 minutes after intake). 


\section{Discussion}

The most important finding in this study is that one hour after drinking a single cup of coffee, ACh-mediated vasodilatation in the skin is acutely enhanced. This suggests that caffeinated coffee acutely augments microvascular reactivity. At the same time, cutaneous vascular conductance in the fingertip is substantially reduced whereas it is unchanged in the skin of forearm.

\section{Plasma caffeine levels}

Drinking a single cup of caffeinated coffee resulted in caffeine plasma concentrations ranging from 0.74 to $1.69 \mu \mathrm{g} / \mathrm{mL}$ after 60 minutes, while concentrations after drinking decaffeinated coffee ranged between 0 and $0.77 \mu \mathrm{g} / \mathrm{mL}$. We did not register how much coffee the subjects consumed regularly, but we know that the majority of the subjects were regular coffee drinkers. Despite the fact that subjects were asked to refrain from the ingestion of any caffeine containing products for at least 24 hours before the experiment, the plasma caffeine levels measured after intake of decaffeinated coffee can be explained by earlier intake of coffee or other caffeine containing products by the subjects. Caffeine is almost completely absorbed within 45 minutes of ingestion [26,27]. The mean half-life of caffeine in plasma of healthy subjects is about 5 hours, although it may vary between 2 and 8 hours as a result of innate individual differences, as well as physiological and environmental factors influencing the metabolism of caffeine. In particular, the use of oral contraceptives is known to increase caffeine plasma half-life [28], whereas it does not seem to be dependent on the menstrual cycle [29]. 


\section{Blood pressure and heart rate}

In most studies coffee drinking acutely raised systolic and diastolic blood pressure. The pressor effect of coffee is believed to be caused by caffeine, which inhibits adenosine receptors and increases circulating norepinephrine concentrations. A dose of 200-250 mg of caffeine has been found to increase systolic blood pressure by 3-14 mmHg and diastolic blood pressure by 4-13 mmHg in normotensive subjects, with a maximum effect reached after 60 minutes [11]. Based on these findings, we hypothesized that blood pressure would increase by 1-4 mmHg after intake of caffeinated coffee in our study. Instead, blood pressure decreased slightly during the first 60 minutes, both after caffeinated and decaffeinated coffee intake, although this decrease was more pronounced and reached significance after intake of decaffeinated coffee. The small decrease in blood pressure observed during the experiment can be explained by the fact that subjects were lying semi-supine throughout the experiment and may not have been completely hemodynamically acclimatized when the baseline blood pressure was measured. Possibly, the caffeine content used in our study was too low to cause a measurable effect on blood pressure with the number of subjects included in the current study, in agreement with a previous study in which no significant increase in peripheral systolic blood pressure was found after intake of coffee containing $80 \mathrm{mg}$ of caffeine [12]. Also, caffeine increases blood pressure more in hypertensive and older subjects, compared to normotensive, younger subjects, that were included in our study [30,31].

Yet another possible explanation for the absence of an increase in blood pressure is that at least some of the subjects were regular coffee drinkers. Evidence on whether or not the pressor effect of caffeine is affected by tolerance is conflicting, although some studies have presented a decreased pressor responses within a few days of the start of regular intake of caffeine. In other studies, however, caffeine was still able to elevate blood pressure during 
habitual consumption. Moreover, the development of tolerance to the pressor effect of caffeine may vary between individuals [11].

\section{Effects of coffee on microvascular function}

This is the first study in which the effects of coffee on endothelium-dependent vasodilation observed after iontophoresis of ACh and SNP were investigated. We found that this response was significantly stronger when subjects had ingested caffeinated coffee, compared to the response after ingesting decaffeinated coffee. Although the vasodilatory response to SNP was stronger after drinking caffeinated coffee, this effect was not significant. When ACh binds to muscarinic receptors of endothelial cells, they release nitric oxide, causing relaxation of smooth muscle cells. Iontophoresis of ACh in the skin has therefore become an established method to assess endothelial function. Other mechanisms have also been suggested to be involved in the microvascular response to ACh in the skin, including stimulation of prostaglandins and endothelium-derived hyperpolarizing factor [32-34]. SNP is an NO donor that directly causes smooth muscle relaxation independent of endothelial function. Because the iontophoretic current in itself can cause vasodilatation $[35,36]$ in the absence of any drug effect, the nonspecific vasodilatory response is often assessed using iontophoresis of $\mathrm{NaCl}$. Although there are not many studies that specifically have investigated the microvascular effects of coffee intake in the skin, our findings are largely in agreement with previous findings regarding effects of coffee or caffeine on general endothelial function. In the isolated rat aorta, caffeine caused endothelium-dependent relaxation through release of calcium ions from the endoplasmatic reticulum and the suppression of degradation of cyclic guanosine monophosphate, as well as endothelium-independent vasodilatation through suppression of cAMP degradation [16,37] found that $300 \mathrm{mg}$ caffeine increased forearm blood flow in response to intra-arterial infusion of ACh, which could be reversed by co-infusion of the nitric 
oxide synthase inhibitor L-NMMA, suggesting an involvement of nitric oxide. Likewise, intake of $200 \mathrm{mg}$ caffeine capsules improved endothelial function assessed by brachial artery flow-mediated dilatation (FMD) in subjects with and without coronary artery disease [17]. In women with type-2 diabetes, coffee consumption was inversely associated with plasma markers of inflammation and endothelial dysfunction [38]. In contrast, at least two studies have found an acute impairing effect of coffee on endothelial function when assessed using flow-mediated dilatation [14,15]. In both studies, subjects were studied after an overnight fast, whereas in our study, subjects were allowed to eat on the morning of the experiment. Polyphenols in coffee may improve postprandial hyperglycemia and endothelial function $[39,40]$ and a synergistic effect between caffeine and these antioxidants may have caused the improvement in endothelial function that we found in our study. Also, in these contrasting studies, responses were measured in the brachial artery, which is a large conducting vessel, whereas we studied responses in the skin microcirculation. Indeed, augmenting effects on endothelial function have mainly been found in the microcirculation, whereas impairing effects have been observed in large conduit vessels [18]. Finally, differences in brewing methods and in concentration of various active ingredients in coffee may exist between studies. The findings in this study, together with the findings from previous studies, however suggest that intake of caffeinated coffee has an augmenting effect on endothelium-dependent microvascular function, with involvement of the nitric oxide pathway.

The PORH response in the forearm skin was not affected by the intake of caffeinated coffee or decaffeinated coffee. In the skin, the PORH response is dependent on sensory nerves and hyperpolarizing mechanisms involving cytochrome epoxygenase metabolites and BKCa channels, whereas prostanoids and nitric oxide are not involved [41,42]. It is noteworthy that the mechanisms in the response in the skin are different from the mechanisms involved in 
reactive hyperemia in larger conductance vessels such as the radial or brachial arteries, which is largely dependent on nitric oxide and endothelium derived hyperpolarizing factor $[43,44]$. Our findings therefore indicate that sensory nerves and hyperpolarizing mechanisms are not affected by intake of either caffeinated coffee or decaffeinated coffee. This is consistent with our finding that the vasodilatory response to iontophoresis of $\mathrm{NaCl}$ was not different after drinking caffeinated coffee and decaffeinated coffee, as this response also is mediated by activation of local sensory nerves. On the other hand, our findings disagree with those of Noguchi et al. [18] who found an improvement in PORH response in the finger after intake of caffeinated coffee. There were however a number of differences between their study and ours. Their study population consisted of healthy, Japanese subjects, whereas ours were healthy Swedish subjects and genetic differences in the ability to metabolize caffeine may exist between populations [45]. Furthermore, the plasma caffeine levels reached in our study were lower than in theirs and they used an occlusion time of 1 minute, measuring blood flow in the finger, whereas we occluded the upper arm for 5 minutes.

\section{Blood flow in forearm skin and fingertip}

Blood flow in the forearm skin was not different before and 45 minutes after intake of either caffeinated coffee or decaffeinated coffee. Within the first hour after intake of a single cup of caffeinated coffee, however, a substantial vasoconstriction in the microvascular bed of the fingertip was observed, about two to three times as strong as the decrease seen after intake of decaffeinated coffee. This is in agreement with previous findings in which caffeinated coffee intake also significantly reduced finger blood flow [18].

In vitro studies have shown that caffeine produces transient contractions of arterial smooth muscle, and contractions on the venous side, caused by changes in intracellular calcium concentrations [11]. Furthermore, caffeine acts as a competitive inhibitor of the 
adenosine receptors of type A1, A2a, and b [46]. Since stimulation of adenosine receptors induces vasodilatation, caffeine may shift the balance between vasodilatory and contractile mechanisms, causing increased vasoconstriction. Thus, the fact that caffeinated coffee caused a net vasoconstriction in the fingertip can be the result of both a direct effect of caffeine on smooth muscle cells, and a direct effect via inhibition of adenosine receptors. The microvascular beds of the fingertip and the forearm differ in various regards, including the capillary density, the heterogeneity and the density of arteriovenous anastomoses. Also, there are differences in measurement depth between LDF and LSCI. These facts may explain why vasoconstriction was observed in the finger tip but not in the forearm.

It should be noted that, in contrast with other studies, we did not observe an increase in blood pressure after subjects ingested caffeinated coffee. Thus, the vasoconstriction response in the fingertip after drinking caffeinated coffee manifests itself in the absence of any systemic changes in blood pressure.

\section{Strengths and limitations}

The main strengths of this study are its design and the methods used to assess microvascular function. We chose to study the effects of drinking a single cup of coffee instead of high doses or pure caffeine, so that any findings are relevant with respect to typical coffee consumption. We eliminated the influence of many confounding variables by using a double blind, randomized, placebo controlled crossover trial. We used LSCI for the measurement of microvascular responses in the skin, which enables measurement of blood flow in a large skin area with high spatial and temporal resolution $[21,22]$. Important with regards to the crossover design in this study, the technique has excellent day-to-day reproducibility, which has been attributed to the fact that blood flow changes can be assessed in an area instead of in a single point. 
The study population consisted of 16 healthy subjects, and we have to acknowledge the fact that some effects could have gone undetected because of the limited power of the study. We can particularly not rule out that the some of the studied effect parameters would have reached significance if a larger number of subjects had been included in the study. The addition of a water control would have resulted in a more complete understanding of the effects of other constituents in coffee, although it would have been impossible to study this in a doubleblinded fashion.

Finally, this cohort of young medical students may be healthier than subjects in previous studies and included both coffee abstainers and habitual coffee drinkers.

\section{Conclusion}

To conclude, the findings in this study suggest that caffeine, ingested through drinking a single cup of caffeinated coffee causes improved endothelium-dependent microvascular reactivity within an hour after intake. Also, caffeine in coffee acutely reduces basal blood flow in the fingertip. 


\section{Highlights}

- Although the long-term effects of coffee consumption on cardiovascular health have been studied extensively, the short-term effects of coffee on microvascular and endothelial function have shown contradicting results.

- One hour after drinking a single cup of coffee, endothelium-dependent vasodilatation in the skin is acutely enhanced, while blood flow in the fingertip is substantially reduced.

- These findings suggest that intake of caffeine through drinking caffeinated coffee acutely augments microvascular reactivity. 


\section{Acknowledgements}

We thank Rasmus Fredriksson for his help with the experiments.

\section{Disclosure}

None

\section{Funding information}

This study was financially supported by ALF grants, Region Östergötland, Sweden. 


\section{References}

1. Riksen NP, Rongen GA, Smits P. Acute and long-term cardiovascular effects of coffee: implications for coronary heart disease. Pharmacol Ther 121: 185-191, 2009.

2. van Dam RM, Willett WC, Manson JE, Hu FB. Coffee, caffeine, and risk of type 2 diabetes: a prospective cohort study in younger and middle-aged U.S. women. Diabetes Care 29: 398-403, 2006.

3. Williams CJ, Fargnoli JL, Hwang JJ, van Dam RM, Blackburn GL, Hu FB, Mantzoros CS. Coffee consumption is associated with higher plasma adiponectin concentrations in women with or without type 2 diabetes: a prospective cohort study. Diabetes Care 31: 504-507, 2008.

4. Siasos G, Oikonomou E, Chrysohoou C, Tousoulis D, Panagiotakos D, Zaromitidou M, Zisimos K, Kokkou E, Marinos G, Papavassiliou AG, Pitsavos C, Stefanadis C. Consumption of a boiled Greek type of coffee is associated with improved endothelial function: the Ikaria study. Vasc Med 18: 55-62, 2013.

5. Baratloo A, Rouhipour A, Forouzanfar MM, Safari S, Amiri M, Negida A. The Role of Caffeine in Pain Management: A Brief Literature Review. Anesth Pain Med 6: e33193, 2016.

6. Li X, Cornelis MC, Liang L, Song F, De Vivo I, Giovannucci E, Tang JY, Han J. A genome-wide analysis of gene-caffeine consumption interaction on basal cell carcinoma. Carcinogenesis 37: 1138-1143, 2016.

7. Liu J, Shen B, Shi M, Cai J. Higher Caffeinated Coffee Intake Is Associated with Reduced Malignant Melanoma Risk: A Meta-Analysis Study. PLoS One 11: e0147056, 2016.

8. O'Keefe JH, Bhatti SK, Patil HR, DiNicolantonio JJ, Lucan SC, Lavie CJ. Effects of habitual coffee consumption on cardiometabolic disease, cardiovascular health, and all-cause mortality. J Am Coll Cardiol 62: 1043-1051, 2013.

9. Freedman ND, Park Y, Abnet CC, Hollenbeck AR, Sinha R. Association of coffee drinking with total and cause-specific mortality. N Engl J Med 366: 1891-1904, 2012.

10. Malerba S, Turati F, Galeone C, Pelucchi C, Verga F, La Vecchia C, Tavani A. A metaanalysis of prospective studies of coffee consumption and mortality for all causes, cancers and cardiovascular diseases. Eur J Epidemiol 28: 527-539, 2013.

11. Nurminen ML, Niittynen L, Korpela R, Vapaatalo H. Coffee, caffeine and blood pressure: a critical review. Eur J Clin Nutr 53: 831-839, 1999.

12. Karatzis E, Papaioannou TG, Aznaouridis K, Karatzi K, Stamatelopoulos K, Zampelas A, Papamichael C, Lekakis J, Mavrikakis M. Acute effects of caffeine on blood pressure and wave reflections in healthy subjects: should we consider monitoring central blood pressure? Int J Cardiol 98: 425-430, 2005. 
13. Greer F, Hudson R, Ross R, Graham T. Caffeine ingestion decreases glucose disposal during a hyperinsulinemic-euglycemic clamp in sedentary humans. Diabetes 50: 2349-2354, 2001.

14. Papamichael CM, Aznaouridis KA, Karatzis EN, Karatzi KN, Stamatelopoulos KS, Vamvakou G, Lekakis JP, Mavrikakis ME. Effect of coffee on endothelial function in healthy subjects: the role of caffeine. Clin Sci (Lond) 109: 55-60, 2005.

15. Buscemi S, Verga S, Batsis JA, Donatelli M, Tranchina MR, Belmonte S, Mattina A, Re A, Cerasola G. Acute effects of coffee on endothelial function in healthy subjects. Eur J Clin Nutr 64: 483-489, 2010.

16. Umemura T, Ueda K, Nishioka K, Hidaka T, Takemoto H, Nakamura S, Jitsuiki D, Soga J, Goto C, Chayama K, Yoshizumi M, Higashi Y. Effects of acute administration of caffeine on vascular function. Am J Cardiol 98: 1538-1541, 2006.

17. Shechter M, Shalmon G, Scheinowitz M, Koren-Morag N, Feinberg MS, Harats D, Sela BA, Sharabi Y, Chouraqui P. Impact of acute caffeine ingestion on endothelial function in subjects with and without coronary artery disease. Am J Cardiol 107: 1255-1261, 2011.

18. Noguchi K, Matsuzaki T, Sakanashi M, Hamadate N, Uchida T, Kina-Tanada M, Kubota H, Nakasone J, Sakanashi M, Ueda S, Masuzaki H, Ishiuchi S, Ohya Y, Tsutsui M. Effect of caffeine contained in a cup of coffee on microvascular function in healthy subjects. $J$

Pharmacol Sci 127: 217-222, 2015.

19. Bonita JS, Mandarano M, Shuta D, Vinson J. Coffee and cardiovascular disease: in vitro, cellular, animal, and human studies. Pharmacol Res 55: 187-198, 2007.

20. Buscemi S, Batsis JA, Arcoleo G, Verga S. Coffee and endothelial function: a battle between caffeine and antioxidants? Eur J Clin Nutr 64: 1242-1243, 2010.

21. Roustit M, Millet C, Blaise S, Dufournet B, Cracowski JL. Excellent reproducibility of laser speckle contrast imaging to assess skin microvascular reactivity. Microvasc Res 80: 505511, 2010.

22. Iredahl F, Lofberg A, Sjoberg F, Farnebo S, Tesselaar E. Non-Invasive Measurement of Skin Microvascular Response during Pharmacological and Physiological Provocations. PLoS One 10: e0133760, 2015.

23. Serne EH, Stehouwer CD, ter Maaten JC, ter Wee PM, Rauwerda JA, Donker AJ, Gans RO. Microvascular function relates to insulin sensitivity and blood pressure in normal subjects. Circulation 99: 896-902, 1999.

24. Ijzerman RG, Serne EH, van Weissenbruch MM, de Jongh RT, Stehouwer CD. Cigarette smoking is associated with an acute impairment of microvascular function in humans. Clin Sci (Lond) 104: 247-252, 2003.

25. Ramalakshmi K, Raghavan B. Caffeine in coffee: its removal. Why and how? Crit Rev Food Sci Nutr 39: 441-456, 1999. 
26. Bonati M, Latini R, Galletti F, Young JF, Tognoni G, Garattini S. Caffeine disposition after oral doses. Clin Pharmacol Ther 32: 98-106, 1982.

27. Liguori A, Hughes JR, Grass JA. Absorption and subjective effects of caffeine from coffee, cola and capsules. Pharmacol Biochem Behav 58: 721-726, 1997.

28. Patwardhan RV, Desmond PV, Johnson RF, Schenker S. Impaired elimination of caffeine by oral contraceptive steroids. J Lab Clin Med 95: 603-608, 1980.

29. Kamimori GH, Joubert A, Otterstetter R, Santaromana M, Eddington ND. The effect of the menstrual cycle on the pharmacokinetics of caffeine in normal, healthy eumenorrheic females. Eur J Clin Pharmacol 55: 445-449, 1999.

30. Hartley TR, Sung BH, Pincomb GA, Whitsett TL, Wilson MF, Lovallo WR. Hypertension risk status and effect of caffeine on blood pressure. Hypertension 36: 137-141, 2000.

31. Izzo JL, Jr., Ghosal A, Kwong T, Freeman RB, Jaenike JR. Age and prior caffeine use alter the cardiovascular and adrenomedullary responses to oral caffeine. Am J Cardiol 52: 769-773, 1983.

32. Durand S, Tartas M, Bouye P, Koitka A, Saumet JL, Abraham P. Prostaglandins participate in the late phase of the vascular response to acetylcholine iontophoresis in humans. J Physiol 561: 811-819, 2004.

33. Gaubert ML, Sigaudo-Roussel D, Tartas M, Berrut G, Saumet JL, Fromy B. Endotheliumderived hyperpolarizing factor as an in vivo back-up mechanism in the cutaneous microcirculation in old mice. J Physiol 585: 617-626, 2007.

34. Morris SJ, Shore AC. Skin blood flow responses to the iontophoresis of acetylcholine and sodium nitroprusside in man: possible mechanisms. J Physiol 496 ( Pt 2): 531-542, 1996.

35. Grossmann M, Jamieson MJ, Kellogg DL, Jr., Kosiba WA, Pergola PE, Crandall CG, Shepherd AM. The effect of iontophoresis on the cutaneous vasculature: evidence for currentinduced hyperemia. Microvasc Res 50: 444-452, 1995.

36. Tesselaar E, Sjoberg F. Transdermal iontophoresis as an in-vivo technique for studying microvascular physiology. Microvasc Res 81: 88-96, 2011.

37. Hatano Y, Mizumoto K, Yoshiyama T, Yamamoto M, Iranami H. Endothelium-dependent and -independent vasodilation of isolated rat aorta induced by caffeine. Am J Physiol 269: H1679-1684, 1995.

38. Lopez-Garcia E, van Dam RM, Qi L, Hu FB. Coffee consumption and markers of inflammation and endothelial dysfunction in healthy and diabetic women. Am J Clin Nutr 84: 888-893, 2006.

39. Jokura H, Watanabe I, Umeda M, Hase T, Shimotoyodome A. Coffee polyphenol consumption improves postprandial hyperglycemia associated with impaired vascular endothelial function in healthy male adults. Nutr Res 35: 873-881, 2015. 
40. Ochiai R, Sugiura Y, Otsuka K, Katsuragi Y, Hashiguchi T. Coffee bean polyphenols ameliorate postprandial endothelial dysfunction in healthy male adults. Int J Food Sci Nutr 66: 350-354, 2015.

41. Cracowski JL, Gaillard-Bigot F, Cracowski C, Sors C, Roustit M, Millet C. Involvement of cytochrome epoxygenase metabolites in cutaneous postocclusive hyperemia in humans. $J$ Appl Physiol (1985) 114: 245-251, 2013.

42. Lorenzo S, Minson CT. Human cutaneous reactive hyperaemia: role of BKCa channels and sensory nerves. J Physiol 585: 295-303, 2007.

43. Mullen MJ, Kharbanda RK, Cross J, Donald AE, Taylor M, Vallance P, Deanfield JE, MacAllister RJ. Heterogenous nature of flow-mediated dilatation in human conduit arteries in vivo: relevance to endothelial dysfunction in hypercholesterolemia. Circ Res 88: 145-151, 2001.

44. Bellien J, Iacob M, Gutierrez L, Isabelle M, Lahary A, Thuillez C, Joannides R. Crucial role of $\mathrm{NO}$ and endothelium-derived hyperpolarizing factor in human sustained conduit artery flow-mediated dilatation. Hypertension 48: 1088-1094, 2006.

45. Cornelis MC, El-Sohemy A, Kabagambe EK, Campos H. Coffee, CYP1A2 genotype, and risk of myocardial infarction. JAMA 295: 1135-1141, 2006.

46. Shaughness G, Akelina Y, Strauch RJ. Dietary guidelines for caffeine and chocolate after digital replantation. J Hand Surg Am 40: 810-812, 2015. 